Journal of Computer Science 5 (4): 297-301, 2009

ISSN 1549-3636

(C) 2009 Science Publications

\title{
Optimal Power Control with Overhearing Avoidance for Wireless Sensor Networks
}

\author{
M. Ramakrishnan and P. Vanaja Ranjan \\ Department of Electrical and Electronics Engineering, \\ College of Engineering, Guindy, Anna University, Chennai, India
}

\begin{abstract}
Problem statement: Extending lifetime of the battery operated wireless sensor nodes through the design of low power medium access control protocols dealt in this study. Approach: In this study energy efficient Optimal Power Control MAC with Overhearing Avoidance (OPC-OA) was proposed. The transmission power of every node was dynamically changed for the optimal connectivity between nodes. The optimal transmission power for a link was estimated in this OPC-AC algorithm by measuring the link quality by using RSSI. The energy consumption analysis of proposed MAC had been done in MATLAB based discrete event simulation. Results: The comparison of energy consumption analysis of the proposed MAC with the On Demand Transmission Power Control (ODTPC) had been done. Conclusion: The results showed the proposed transmission power control MAC with overhearing avoidance outperforms the On Demand Transmission Power Control (ODTPC) in terms of energy consumption.
\end{abstract}

Key words: Optimal power control, transmission power control, wireless sensor networks, overhearing avoidance, energy efficient MAC

\section{INTRODUCTION}

Wireless sensor network is the wireless network of tiny devices which has both sensing and communication capabilities. Conserving power in this battery operated tiny sensor nodes is a key challenge in the protocol design for the wireless sensor networks. The major proportion of the power consumed by the node is for its radio transmission and reception. A typical RF transceiver consumes $21 \mathrm{~mA}$ of current (at $10 \mathrm{dBm}$ ) in transmission mode and $18 \mathrm{~mA}$ of current in the reception mode ${ }^{[3]}$. So protocols are designed to optimize the number of bytes transmitted/received in the network for the intended communication. Often energy efficiency is dealt in the Medium Access Control (MAC) layer in the protocol for wireless sensor network by introducing sleep schedule. That is the node will keep its radio off for much time and listens and transmit in the wireless medium for a little while. This kind of sleep scheduling causes a problem of increased end to end latency. Another way of reducing the energy consumption without increasing the end to end latency is the Transmission power control. Nowadays many transceivers have the ability to change their transmission power dynamically by changing the amplifier gain setting. By exploiting this flexibility, the transmission power of a link between two nodes can be adaptively varied to keep the optimal connectivity between the nodes. Reducing the transmission power not only gives the minimum power consumption but gives minimum interference in the network.

The earlier works are done which estimates the minimum transmission power which is sufficient to have full connectivity in the network, is assigned to all the nodes in the network. But with the rapid growth in the Integrated Circuit technology, it is possible to have a simple RF transceiver which still has dynamic transmission power control capability. In this study an Optimal Power Control with Overhearing Avoidance Medium Access Control (OPC-OA-MAC) protocol is designed which exploits the flexibility of changing the transmission power offered by the modern RF transceivers.

In Shan $\operatorname{Lin}^{[4]}$ and Jingbin Zhang et al. ${ }^{[2]}$ proposes Adaptive Transmission Power Control (ATPC) in which a predictive model is designed to find the correlation between the transmission power and the link quality. $\mathrm{In}^{[5]}$ two transmission power control algorithms are simulated and it is also shown that these protocols outperform the fixed power assignment protocols. In study, Alaa Muqattash and Marwan Krunz ${ }^{[6]}$ proposes a Power Control Dual Channel Protocol (PCDC) which

Corresponding Author: M. Ramakrishnan, Department of Electrical and Electronics Engineering, College of Engineering, Guindy Anna University, Chennai, India 
uses one channel for control packet (RTS/CTS) transmission with Maximum power and another channel for data packet transmission with less power sufficient to maintain connectivity between nodes. In $^{[7]}$ the empirical analysis of power control algorithms has been done and the reduction in the power consumption has been achieved and it is also stated that the combination of low power MAC design with the power control will yield better energy conservation. It is stated that the asymmetry in the transmission floor will cause the collision of packets. $\operatorname{In}^{[8]}$ it is reported that the variable range power control is superior to common range power control. It is also stated that Variable range power control outperforms the common range power control in energy consumption and in the traffic carrying capacity of a node. $\operatorname{In}^{[9]}$, a power controlled multiple access protocol has been proposed with data and busy tone channel to reduce the floor acquisition done with RTS/CTS exchange. It is also stated that PCMA improves the throughput by two times compared to 802.11 MAC and it is also stated that the performance of PCMA increases when the average distance between the transmitter and receiver decreases. $\mathrm{In}^{[10-11]}$ a power control with blacklisting algorithm has been proposed for wireless sensor networks. PRR is used as a link quality metric and the node will transmit packets at different transmission power level to find the optimal power level. This will obviously reduce the lifetime of the node and in the dense deployment of the wireless sensors: Calculation of PRR will become difficult due to collision. $\mathrm{In}^{[1]}$, in On demand Transmission Power Control (ODTPC) the node, that wants to transmit the packet, checks its neighbor table for the optimal transmission power. If the neighbor table does not contain the optimal power, then the data packets are transmitted at the maximum power and the receiver measures the RSSI. The receiver calculates the optimal power and the RSSI are sent back to the transmitter with acknowledgment. The performance of the ODTPC is compared with that of PCBL and ATPC and it is also shown that ODTPC outperforms these two power control algorithm.

In this study the energy consumption analysis of our proposed OPC-OA MAC has been done and it is compared with that of ODTPC.

\section{MATERIALS AND METHODS}

Optimal Power Control MAC with Overhearing Avoidance: This OPC-OA-MAC is an extension of CSMA/CA with RTS/CTS. OPC-OA-MAC includes the adaptive transmission power control without using separate control channel as in $\mathrm{PCDC}^{[6]}$.
In a simple CSMA/CA protocol node senses whether the medium is idle or not. If the medium is idle, then it can do the transmission of RTS. After getting this RTS control packet the intended receiver sends the CTS. These RTS/CTS control packet flow is to avoid hidden and exposed terminal problem which is prevalent in wireless MAC.

In OPC-OA-MAC the adaptive transmission power control is included as below:

- Sender node senses the carrier. If it is idle, it checks its neighbor table to find the optimal transmission power. If the table doesn't have a valid entry, then RTS is transmitted at maximum power. Otherwise RTS will be transmitted at optimal power

- While receiving the RTS, the intended receiver measures the RSSI by which it can measure the range (d) approximately. Then it makes the entry of the distance metric in the neighbor table

- If the Signal to Noise Ratio (SNR) value is more than the SNR threshold value then the optimal transmission power for the receiver is chosen by using the radio model

- The power level is increased to an extent to compensate for the indoor attenuation factors. Then the intended receiver sends CTS to the sender with the estimated optimal power and this packet contains the power level at which the receiver transmits

- The unintended nodes receiving the RTS, goes to sleep state for the CTS/DATA/ACK transfer time to avoid overhearing

- Sender also changes its power level and does the data packet transfer at reduced power which depends upon the range between the transmitter and receiver. The sender also updates the neighbor table

In OPC-OA MAC all the packets have a field to indicate the power level at which it gets transmitted. This kind of power control not only alleviates huge power consumption but also the interference problem and facilitates the spatial reuse of the channel.

Simulation of OPC-OA MAC: A discrete event simulation program has been written in MATLAB to analyze the energy consumption for the proposed Optimal Power Control with Overhearing Avoidance Medium Access Control protocol. As the single hop wireless networking scenario is considered, Physical layer and MAC layer have been coded. In this simulation an Ideal Power Consumption (IPC) has been 
assumed in which the channel gain is considered as a constant and known. So the optimal power required can be computed exactly. The free space model is used as the radio model and in MAC layer CSMA/CA with RTS/CTS and Optimal Power Control with Overhearing Avoidance has been implemented. The simulation parameters are shown in the following Table 1.

Star topology is taken with a single sink node in the center and multiple source nodes around the center with equal distance from the sink node. The topologies taken are shown in Fig. 1 and 2.

For traffic pattern, multiple source and a single sink has been considered in this simulation. The traffic is defined as follows. All the source nodes give a packet to sink node. The simulation has been repeated for various distances between the source nodes and sink node and the transmission energy consumption for various distances has been reported.

Table 1: Simulation parameters

\begin{tabular}{lll}
\hline Sr. No. & Simulation parameters & Value \\
\hline 1 & Frequency & $433.92 \mathrm{MHz}$ \\
2 & Radio model & Free space model \\
3 & MAC layer & CSMA/CA with \\
& & RTS/CTS and OPC \\
4 & Data rate & $9600 \mathrm{bps}$ \\
5 & Max. power & $+13 \mathrm{dBm}$ \\
6 & Area & $100 \times 100 \mathrm{~m}$ \\
7 & Topology & Single hop \\
8 & SNR $_{\text {threshold }}$ & $+30 \mathrm{dBm}$ \\
9 & Size of packets: & $7 / 7 / 50 / 7$ Bytes \\
& RTS/CTS/DATA/ACK & \\
\hline
\end{tabular}

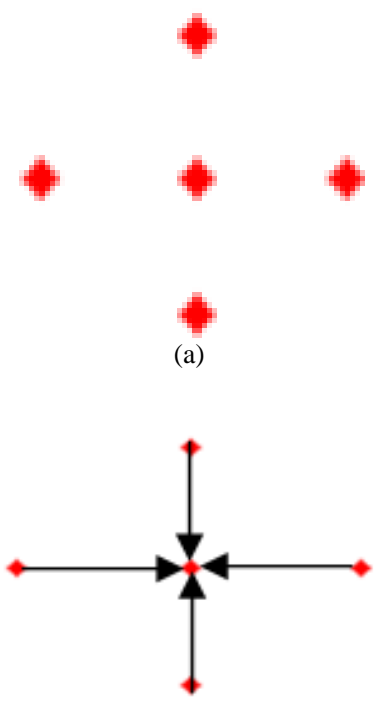

(b)

Fig. 1: Five node star topologies; (a): Distance $5 \mathrm{~m}$; (b): Distance $10 \mathrm{~m}$
An energy model has been developed to calculate the energy consumption of PICSENSE node at various transmission power levels. The PICSENSE node has PIC18F4620, an 8 bit microcontroller and ADF7020-1, Analog Devices short range $\mathrm{RF}$ transceiver. The transmission power of ADF7020-1 can be programmed to have the transmission power in 64 steps from -16 to $+13 \mathrm{dBm}$. As PICSENSE node uses ADF7020-1 RF transceiver, the relation between the Transmission power levels and the current consumption has been interpolated from the data given $\mathrm{in}^{[3]}$. A linear relationship between the power level and the corresponding current consumption is given in the following expression:

$$
\mathrm{C}_{\mathrm{i}}=\left(0.8 \mathrm{P}_{\mathrm{i}}+12.667\right) \mathrm{mA}
$$

Where:

$\mathrm{C}_{\mathrm{i}}=$ Current consumption for ith packet transmission.

$\mathrm{P}_{\mathrm{i}}=$ Transmission power for ith packet transmission (in $\mathrm{dBm}$ )

Total Energy Consumption (TEC) of the wireless sensor network can be computed by the following expression:

$$
\mathrm{TEC}=\mathrm{E}_{\text {processor }}+\mathrm{E}_{\mathrm{Rx}}+\mathrm{E}_{\mathrm{Tx}}
$$

where, energy spent by the processor in nodes:

$$
\mathrm{E}_{\text {processor }}=\mathrm{N}_{\mathrm{n}} * \mathrm{P}_{\text {processor }} * \text { Total_Time }
$$

Energy spent when transceivers are in reception mode:

$$
\mathrm{E}_{\mathrm{Rx}}=\mathrm{N}_{\mathrm{n}} * \mathrm{P}_{\mathrm{Rx}} *\left(\text { Total_Time }-\mathrm{Tx} \_ \text {Time }- \text { Idle_Time }\right)
$$

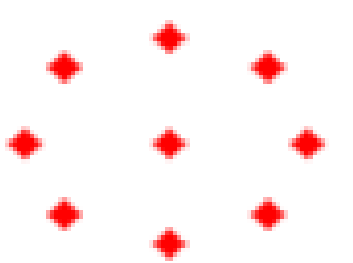

(a)

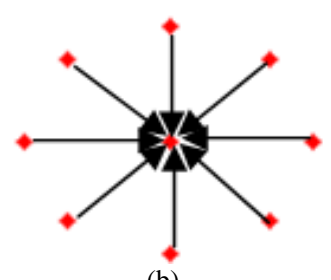

(b)

Fig. 2: Nine node star topologies; (a): Distance $5 \mathrm{~m}$; (b): Distance $10 \mathrm{~m}$ 
For $\mathrm{N}$ packet transmission the transmission energy consumption is modeled as follows:

$$
\mathrm{E}_{\mathrm{Tx}}=\sum_{\mathrm{i}=1}^{\mathrm{i}=\mathrm{N}} 3 \mathrm{C}_{\mathrm{i}} \mathrm{P}_{\mathrm{si}}(16 / \text { Data_rate })
$$

$\mathrm{P}_{\mathrm{si}}=$ Packet size for ith packet

$\mathrm{P}_{\text {processor }}$ and $\mathrm{P}_{\mathrm{RX}}$ are the power spent by processor and the transceiver in the Reception mode respectively:

$\mathrm{N}_{\mathrm{n}} \quad=$ The number of nodes in the network

Total_Time $=$ Total simulation time

Tx_Time = Time for which the transceiver in Transmission mode

Idle_Time $=$ Time for which the transceiver is disabled (i.e.) in sleep state

In this energy model power spent by the sensor and the other peripherals of wireless node are not considered.

\section{RESULTS}

The simulation has been repeated for various distances between the source nodes and the sink node and the total energy consumption of various power control algorithms have been reported. From the results it has been observed that the difference in the energy consumption is significant when the number of nodes and the traffic increases. Figure 3 shows the total network energy consumption for various distances from source nodes to sink node. From Fig. 3 it is observed that the energy consumption of the OPC with overhearing avoidance outperforms the On Demand Transmission Power Control (ODTPC) protocol.

The simulation has been done by changing the number of packets transmitted within a specified Time for various distances from the source nodes to sink node. The results show that when the packet rate increases for the OPC with overhearing avoidance, energy consumption decreases.

\section{DISCUSSION}

Figure 3 shows the energy consumption of Optimal Power Control (OPC), OPC with Overhearing Avoidance and On Demand Transmission Power Control (ODTPC) for various distances between the source nodes and sink node. From the results it is observed that there are no significant differences between the energy consumption of the ODTPC and the OPC MAC for various distances. From Fig. 4 it is observed that the

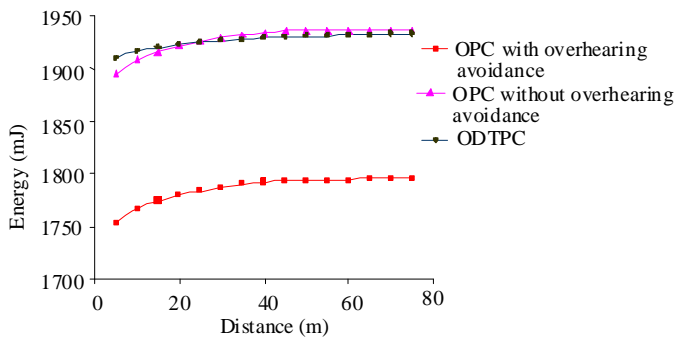

Fig. 3a: Total network energy consumption analysis 5 node topology

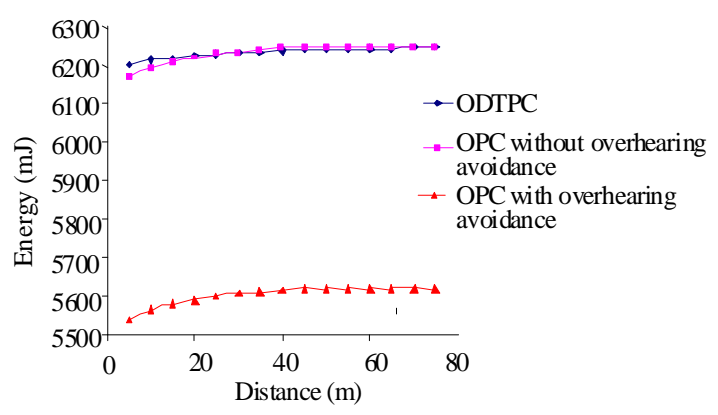

Fig. 3b: Total network energy consumption analysis 9 node topology

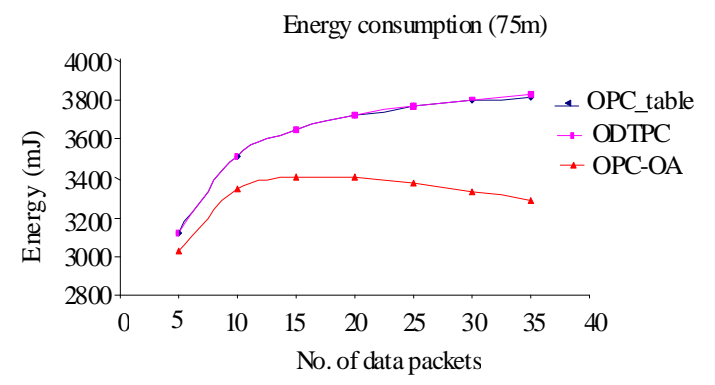

Fig. 4a: Total network energy consumption Vs traffic rate 5 node topology

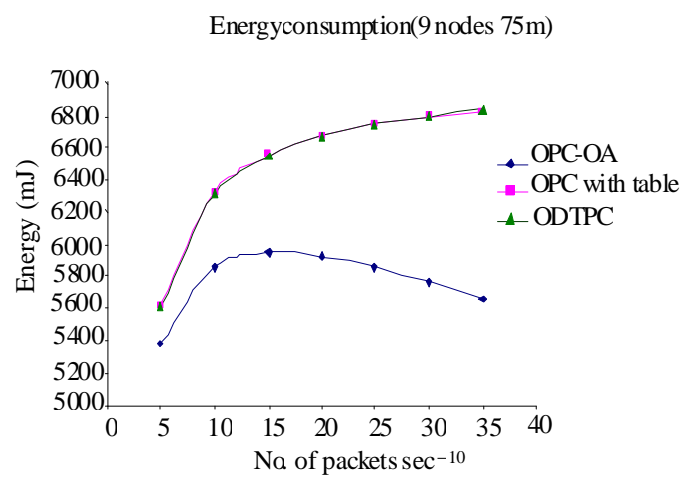

Fig. 4b: Total network energy consumption Vs traffic rate 9 node topology 
difference between the energy consumption of ODTPC and OPC-OA is $7-8 \%$ for the 5 node topology and the difference is $10-11 \%$ for 9 node topology. From this observation it is also obvious that the energy saving by OPC-OA increases when the number of nodes in the network increases.

From the observation from the results shown in Fig. 4, it is observed that when the traffic rate increases the energy consumption by OPC and ODTPC also tends to increase. But when the packet rate increases the energy consumption of OPC-OA tends to decrease as more the unicast packets are transmitted, more will be the idle time for the unintended nodes. This decreases the energy consumption of OPC-OA MAC, when number of unicast packet increases.

\section{CONCLUSION}

In this study an Optimum Power Control with Overhearing Avoidance (OPC-OA) MAC for wireless sensor network is proposed. The proposed OPC-OA MAC has been simulated in MATLAB and the energy consumption analysis of the proposed MAC has been done and the energy consumption of the proposed MAC has been compared with that of the ODTPC protocol. The results show that the OPC-OA outperforms the ODTPC in the energy consumption.

\section{REFERENCES}

1. Kim, J., S. Chang and Y. Kwon, 2008. ODTPC: On demand transmission power control for wireless sensor networks. Proceedings of the International Conference on Information Networking, Jan. 23-25, IEEE Xplore Press, Busan, Korea, pp: 1-5. DOI: 10.1109/ICOIN.2008.4472775

2. Lin, S., J. Zhang, G. Zhou, L. Gu, T. He and J.A. Stankovic, 2006. Adaptive Transmission Power Control (ATPC). Proceedings of the 4th International Conference on Embedded Networked Sensor Systems, Oct. 31-Nov. 03, Boulder, Colorado, USA., pp: 223-236. http://doi.acm.org/10.1145/1182807.1182830

3. Analog Devices 2005. ADF7020-1 Datasheet http://www.analog.com/static/importedfiles/data_sheets/ADF7020-1.pdf

4. Panichpapiboon, S., G. Ferrari and O.K. Tonguz, 2006. Optimal transmit power in wireless sensor networks. IEEE Trans. Mobile Comput., 5: 1432-1447. DOI: 10.1109/TMC.2006.155
5. Kubisch, M., H. Karl, A. Wolisz, L.C. Zhong and J. Rabaey, 2003. Distributed algorithms for transmission power control in wireless sensor networks. Proceeding of the Wireless Communications and Networking Conference, Mar. 20-20, IEEE Xplore Press, New Orleans, LA., USA., pp: 558-563. DOI: 10.1109/WCNC.2003.1200410

6. Muqattash, A. and M. Krunz, 2003. Power Controlled Dual Channel (PCDC) medium access protocol for wireless Ad Hoc networks. Proceedings of the IEEE 22nd Annual Joint Conference of the IEEE Computer and Communications Societies, Mar. 30-Apr. 3, IEEE Xplore Press, USA., pp: 470-480. DOI: 10.1109/INFCOM.2003.1208698

7. Jeong, J., D. Culler and J. Hyuk Oh, 2007. Empirical analysis of transmission power control algorithms for wireless sensor networks. Proceedings of the 4th International Conference on Networked Sensing Systems, June 6-8, IEEE Xplore Press, Braunschweig, pp: 27-34. DOI: 10.1109/INSS.2007.4297383

8. Gomez, J. and R.T. Campbell, 2004. A case for variable-range transmission power control in wireless Ad Hoc networks. Proceedings of the 3rd Annual Joint Conference of the IEEE Computer and Communications Societies, Mar. 7-11, IEEE Xplore Press, USA., pp: 1425-1436. http://ieeexplore.ieee.org/stamp/stamp.jsp?tp=\&isn umber $=$ \&arnumber $=1357027$

9. Monks, J.P., V. Bharghavan and W.M.W. Hwu, 2001. A power controlled multiple accesses for wireless packet networks. Proceedings of the 20th Annual Joint Conference of the IEEE Computer and Communications Societies, Apr. 22-26, IEEE Xplore Press, Anchorage, AK., USA., pp: 219-228. DOI: 10.1109/INFCOM.2001.916704

10. Ramanathan, R. and R. Rosales-Hain, 2000. Topology control of multihop wireless networks using transmit power adjustment. Proceedings of the 9th Annual Joint Conference of the IEEE Computer and Communications Societies, Mar. 2630, IEEE Xplore Press, Tel Aviv, Israel, pp: 404-413. DOI: $10.1109 /$ INFCOM.2000.832213

11. Son, D., B. Krishnamachari and J. Heidemann, 2004. Experimental study of the effects of transmission power control and blacklisting in wireless sensor networks. Proceedings of the 1st Annual IEEE Communications Society Conference on Sensor and Ad Hoc Communications and Networks, Oct. 4-7, IEEE Xplore Press, USA., pp: 289-298. DOI: 10.1109/SAHCN.2004.1381929 